\title{
Human FOXP3+ regulatory T cells in transplantation
}

\author{
Peter Boros ${ }^{1, *}$ and Jonathan S. Bromberg ${ }^{1,2}$ \\ ${ }^{1}$ Recanati/Miller Transplantation Institute, Mount Sinai School of Medicine, New York, NY \\ ${ }^{2}$ Department of Gene and Cell Medicine, Mount Sinai School of Medicine, New York, NY
}

\section{Abstract}

CD4+CD25+FOXP3+ suppressive regulatory T cells (Treg) represent a subset of immune regulatory cells. Based on experimental results, Treg have recently been considered as a potential treatment option in several diseases. Compared with murine Treg, human CD4+CD25+FOXP3+ cells are less well characterized and understood, so a thorough understanding of their biology is vital before clinical applications can be initiated. This review summarizes knowledge on generation, phenotypic characteristics and function of human Treg. The possible role of these cells in organ transplantation, as well as interactions between immunosuppression and Treg are also discussed.

Understanding how excessive or deleterious immune reactions can be suppressed or regulated is as essential as to know how to augment immune responses protective to the host. Suppression of immunity to normal self-constituents in autoimmune diseases, environmental substances in allergy, or microbial components in certain inflammatory diseases, and establishment of immunological tolerance to organ transplants are all pressing clinical needs. Similarly, reversal of the unresponsive state to autologous tumor cells or chronic infections is essential for effective immunotherapy. Thus, regulation of the immune system is a profoundly important key concept in immunology.

The idea of suppressor $\mathrm{T}$ cells is not new, and $\mathrm{T}$ cell mediated suppression of immune responses has attracted vast interest. Many cell types such as CD4+CD25high regulatory $\mathrm{T}$ cells, CD4+ Tr1 cells, CD4+ Th3 cells, CD8+CD28-T cells, CD4-CD8-T cells and NKT cells have each been shown to possess immunoregulatory capacity but the relative importance of these subsets has yet to be defined. The primary focus of this paper will be CD4+CD25+FOXP3+ T cells (Treg). Treg are thought to be important in cancer, autoimmunity, allergy and asthma. Human Treg are significantly less well defined and understood in comparison to their murine counterparts. As generation and modulation of these cells are increasingly considered potential therapeutic modalities, a comprehensive understanding of the biology of human Treg is imperative before clinical applications can be introduced.

In addition to briefly summarizing how Treg may function, we will discuss specific issues related to human Treg such as FOXP3 expression during activation, generation and phenotype. Finally, the review will discuss current knowledge on the possible role of this important subset of cells in organ transplant patients. We will present data on the usefulness monitoring FOXP3 expression in transplant patients, analyze how different immunosuppressive regimens may alter Treg, and examine the possible use of in vitro generated Treg as a treatment modality.

"Corresponding author at: Recanati/Miller Transplantation Institute, Mount Sinai School of Medicine, One Gustave L. Levy Place, POB 1504, New York, NY 10029, T: 212-241-5589, F: 212-426-2233, Peter.Boros@ mountsinai.org. 


\section{Mechanisms of regulation by Foxp3+ T cells}

Foxp3 is a forkhead-winged helix transcription factor gene involved in immune function, and its expression has been used to define this T cell subset. Foxp3 has emerged as the most definitive marker for Treg in mice, and it is crucial to their thymic development and peripheral function. Deficiency and dysfunction of these cells can cause autoimmune disease or elicit tumor immunity. Expansion of their antigen-specific population can establish transplantation tolerance.

Various mechanisms have been proposed to explain suppressive function of Treg on naive or effector T cells. These include interactions between stimulatory (IL-2 and CD28) and inhibitory (glucocorticoid-induced tumor necrosis factor receptor family-related protein (GITR) and cytotoxic T lymphocyte-associated antigen 4 (CTLA-4)) signals, stimulation of dendritic cells via CD80/CD86 molecules, and cell-cell inhibition of effector cells by membrane-bound and soluble factors (e.g., transforming growth factor- $\beta$ (TGF- $\beta$ ) and IL-10) (1). Experimental studies highlighted important aspects of Treg function, such as differences in mechanisms between antigen-specific and non-specific regulation, as well as among pathways involved in vitro versus in vivo. Naturally occurring Treg are antigen nonspecific, while induced Treg are antigen specific in most but not all systems. While in vitro studies primarily suggest a cell-cell contact dependent suppression, in vivo models (e. g., transplantation) emphasize the central role of inhibitory cytokines and surface molecules, and suppression is dependant on a continuous supply of alloantigens (2).

Another possible mechanism is cytokine deprivation-induced apoptosis as shown in a mouse model of inflammatory bowel disease. In addition to apoptosis, cytokine deprivation may interfere with T cell activation or enhance Treg cell suppressive activity (3). Autoreactive B cells specific for tissue autoantigen can also be controlled by Treg. In transgenic mice expressing OVA and HEL in pancreatic islet beta cells, transferred HEL-specific transgenic B cells disappeared within 3 weeks when immunized with the autoantigen. Depletion of CD25+ FoxP3+ cells restored cell numbers suggesting that Treg suppressed autoreactive B cells (4).

In human Treg, additional molecules and pathways associated with suppressor function have been described. A cell surface molecule, called GARP, (or LRRC32), specifically expressed in Treg, is activated through the T cell receptor (TCR). GARP is a leucine-rich repeatcontaining protein, playing a role in protein-protein interactions and signal transduction. GARP over-expression in human naïve $\mathrm{T}$ cells inhibited their proliferation and cytokine secretion, induced expression of FOXP3, and induced partial suppressive function. Additionally, silencing FOXP3 in human Treg cells resulted in reduced expression of GARP and decreased suppressive function (5).

\section{Human Treg - a reversible stage of peripheral differentiation? FOXP3 expression by $T$ cells during activation}

The role and significance of FOXP3 in humans is complicated by the fact that CD4+CD25-FOXP3- T cells can be induced to express FOXP3, and FOXP3 can be upregulated without associated regulatory activity. Virtually all human CD4+CD25-FOXP3$\mathrm{T}$ cells and CD8+CD25-FOXP3- $\mathrm{T}$ cells can attain a transient FOXP3+CD25+ state during activation. In this state, these cells inhibit in vitro proliferation of autologous CD4+CD25- T cells (6).

FOXP3 specific antibodies used in flow cytometric analysis also demonstrated that FOXP3 expression is not unique to a subset of activated cells. Instead, any mode of activation, 
including anti-CD3 monoclonal antibody, or allogeneic or antigen-specific stimulation, lead to a phase of FOXP3 expression in every $\mathrm{T}$ cell $(7,8)$. Some studies have shown rapid downregulation of FOXP3 in activated T cells, questioning whether these cells represent suppressive Treg. Isolating either CD25+ cells or proliferating cells from bulk cultures after activation, suppressive activity was definitively demonstrated in the induced FOXP3+ T cells (9). Thus, transient induction of FOXP3 by activation of human $\mathrm{T}$ cells results in regulatory properties of these cells.

While most activated cells eventually downregulate FOXP3 expression, a small percentage will sustain FOXP3 expression, even 15-20 days later. Transient upregulation of FOXP3 and regulatory function, followed by sustained expression in a smaller portion of cells may be a way of generating Treg in the periphery following antigen stimulation, and to acquire broad specificity for self and foreign antigens.

In addition to FOXP3, Treg have been associated with multiple markers including CD25, CTLA-4, GITR, CD69, CD44 and downregulated CD127 (IL-7 receptor $\alpha$ chain) (6). A recent study has established CD127 as a useful maker for isolating Treg from type 1 diabetic patients (10). No surface or intracellular markers can clearly distinguish Treg in humans, as expression patterns of these markers can be mimicked by activated T cells. Similarly, analysis of microRNAs expressed by regulatory $\mathrm{T}$ cells determined that both Treg and activated $\mathrm{T}$ cells have very similar microRNA expression profiles. Investigation of genome wide targets of FOXP3 using chip assays revealed that FOXP3 represses and activates genes involved in $\mathrm{T}$ cell activation (11). These data demonstrate a close relationship between $\mathrm{T}$ cell activation and acquisition of suppressive capability, suggesting that circulating human Treg may be a subset of activated $\mathrm{T}$ cells.

Recent evidence indicates alternative roles and phenotypes for FOXP3+ T cells. Some FOXP3+ cells are transiently activated effector $\mathrm{T}$ cells, so that regulatory activity and effector function co-exist in the same cell at the same time. Treg plasticity is supported by studies showing that human Treg can differentiate into IL-17-producing cells, and T cells with induced FOXP3+ can secrete effector cytokines, such as TNF $\alpha$ (12). CD4+FOXP3+ T cells that express CCR6, ROR $\gamma \mathrm{t}$ transcription factor, and have the capacity to produce IL-17 can be detected in human blood and lymphoid tissue. These cells strongly inhibit the proliferation of CD4+ responder T cells and maintain their suppressive function by a cell-tocell contact mechanism. As the human thymus does not contain IL-17-producing Treg cells, these IL-17+FOXP3+ Treg must be generated in the periphery (13).

\section{Peripheral versus thymic generation of human Treg}

In mice, many Treg are generated in the thymus, with some developing in the periphery. It seems unlikely that a small subset of similar cells would persist throughout the long lifespan of humans, without either ongoing expansion or peripheral induction of Treg. There is currently no clear marker that can distinguish natural thymically generated from induced Treg in mice or humans.

A recent study analyzed thymic production of FOXP3+ Treg in over 50 patients who had undergone cardiac surgery and thymectomy, and compared it with the level of FOXP3 expression in the blood. Expression in thymic tissue samples appeared to be stable over time. Further, no correlation between thymic and peripheral expression of FOXP3 was found. Monitoring the expression of the cell cycle-associated marker Ki67 revealed that a substantial number of FOXP3 + cells were dividing in the peripheral blood but not in the thymus. Thus, Treg peripheral homeostasis is complex, involves increased proliferation, and does not necessarily reflect thymic production (14). 
Human Treg are a rapidly proliferating population, and are short-lived and undergo apoptosis at much higher rates compared to naïve or memory T cells (15). As Treg numbers do not significantly decline with age, thymic generation or expansion of thymic-derived Treg cannot explain their lifelong persistence. Comparison of V $\beta$ usage of the TCR repertoires of $\mathrm{CD} 4+\mathrm{CD} 45 \mathrm{RO}+\mathrm{CD} 25 \mathrm{hi}$ and $\mathrm{CD} 4+\mathrm{CD} 45 \mathrm{RO}+\mathrm{CD} 25-$ populations showed similarit $y$ between the two populations suggesting that they share the same precursor population (16). Thus, during an antigen-specific response, many activated $T$ cells attain a FOXP3+ state, with the potential of a small subset to persist as Treg. The remaining cells downregulate FOXP3, reside in the memory pool, and repeat this process during re-exposure to the antigen.

\section{FOXP3+ Treg and transplantation}

In transplantation, FOXP3+ T cells play a role in the suppression of donor-activated effector $\mathrm{T}$ cells and in tolerance induction. Human studies investigated FOXP3 expression in graft samples and peripheral blood, but the significance of altered levels or cell numbers are not well understood. In addition to interpreting FOXP3 expression in transplant related events, understanding the impact of immunosuppressive treatment modalities on FOXP3 expression and FOXP3 positive cells remains to be fully explored.

\section{FOXP3+ T cells in organ transplant patients}

Acute rejection-Endomyocardial biopsies taken during acute cellular rejection after cardiac transplantation displayed higher FOXP3 mRNA expression compared to those without histologically proven rejection. When FOXP3 gene expression in the peripheral blood was analyzed, no association with rejection or non-responsiveness was found (17). Similarly, higher FOXP3 mRNA levels in the urine of renal transplant patients with an acute rejection episode were observed, suggesting that FOXP3 mRNA expression is associated with anti-donor immune reactivity (18). When FOXP3 mRNA expression using RT-PCR was analyzed in over 80 renal transplant biopsies, higher mRNA levels were associated with rejection, younger donor age and longer posttransplant period. FOXP3 expression did not correlate with favorable graft outcome (19). Intragraft FOXP3 increased within the first year after liver transplantation, but was not reflected by changes in the blood. Elevated FOXP3 in biopsy tissue correlated with hepatitis $\mathrm{C}$ virus reinfection and previous episodes of acute rejection. The lack of correlation between FOXP3 expression and desirable outcome questions the role of these cells under current immunosuppressive regimens.

Chronic rejection-Chronic rejection is an important cause of long-term graft loss that might be prevented by the induction of tolerance. Renal transplant patients with chronic rejection have lower numbers of peripheral CD4+CD25high T cells than operationally tolerant patients, patients with stable graft function, or healthy volunteers. Investigation of FOXP3 expression in these cells revealed that chronic rejection is associated with a decreased number of $\mathrm{CD} 4+\mathrm{CD} 25$ highFOXP3 $+\mathrm{T}$ cells with normal regulatory profile, whereas graft acceptance is associated with $\mathrm{CD} 4+\mathrm{CD} 25 \mathrm{highFOXP3+T}$ cell numbers similar to healthy volunteers. These data suggest that Treg numbers, rather than their intrinsic suppressive capacity, may contribute to determining the long-term fate of renal transplants (20). Similar reports came from operationally tolerant liver transplant patients: $\mathrm{CD} 4+\mathrm{CD} 25 \mathrm{high}+\mathrm{T}$ cell ratios were increased in the tolerant patients' peripheral blood, and FOXP3 expressing $\mathrm{T}$ cells were also present in the tolerant liver (21).

The presence of Treg seems to correlate with graft acceptance in living donor liver transplantation. FOXP3 mRNA expression and the presence of FOXP3+ CD4 and CD8 cells were analyzed in biopsies from tolerant recipients and compared with recipients on maintenance immunosuppression, grafts removed because of chronic rejection and normal 
liver tissue. FOXP3 mRNA in the biopsies of tolerant patients was higher. Additionally, the number of FOXP3 + CD4 cells was significantly increased in the portal areas in these patients, compared with the other groups (22).

In summary, these clinical data are commensurate with the observations above that most if not all human T cells can transiently express FOXP3, so that monitoring this gene is not the equivalent of monitoring fully suppressive Treg, and the increased FOXP3 expression may only reflect $\mathrm{T}$ cell activation due to other factors (e.g., inflammation). These data also show the differences in gene expression between the graft and blood seen for multiple organ types, underlining the importance of local immune monitoring (23). Furthermore, to establish a reliable correlation between FOXP3 expression and graft acceptance, other confounding factors need to be considered (e.g., immunosuppressive therapy).

\section{Imunosuppression and FOXP3+ Treg}

Recent evidence indicates that several commonly used immunosuppressive drugs have detrimental effects on the induction and function of Treg, whereas other drugs appear to spare these cells or may even promote their function or survival.

In humans, the percentage of circulating CD4+CD25+ and CD4+FOXP3+ cells decreases after transplantation. Reduction of Treg is not limited to peripheral blood; lower FOXP3 mRNA and positive $T$ cells were found normal colon tissue from liver transplant recipients compared with healthy controls (24).

The interleukin-2 receptor $\alpha$ chain plays a major part in shaping immune activation, due to its role in T cell proliferation and survival. Pharmaceutical agents that block the IL-2 pathways blunt effector responses. However, such strategies could adversely affect CD25+FOXP3+ Treg. Cardiac allograft recipients treated with the humanized anti-CD25 antibody Daclizumab displayed FOXP3 expression patterns consistent with functional T regulatory cell populations. High levels of FOXP3 were observed to correlate with lower incidence of acute rejection, as well as lower levels of anti-donor HLA antibody production. Therefore, Treg appear fully functional in patients treated with Daclizumab, even when 5 doses were administered. These results indicated that Daclizumab treatment did not interfere with the generation of Treg (25).

Recently, the effects of Belatacept (CTLA-4Ig) and basiliximab (anti-CD25 $\alpha$ monoclonal antibody) on the Treg in peripheral blood and in kidney biopsies of patients with acute rejection were analyzed. Basiliximab caused a transient loss of both FOXP3+ and FOXP3CD25+ T cells in the circulation. No long-term effect on circulating Treg with combined Belatacept and basiliximab treatment was observed compared to a CNI-treated group of patients. Belatacept-treated patients displayed significant accumulation of FOXP3+ T cells in graft biopsies during acute rejection when compared to CNI-treated patients (26).

Kidney transplant patients receiving CNI maintenance treatment had a significantly lower percentage of peripheral CD4+CD25high T cells compared with patients receiving rapamycin (27). In stable liver transplant recipients reduced numbers of peripheral CD4+CD25highFOXP3+ Treg were associated with high CNI dosing in comparison to low CNI dosing and healthy volunteers. Conversion of kidney transplant recipients from tacrolimus/MMF to rapamycin monotherapy significantly increased the percentage and absolute number of circulating CD4+CD25highFOXP3+ Treg, while conversion to tacrolimus monotherapy did not have this effect. These findings suggested a shift in balance between immune reactive and regulatory cells by either CNI or rapamycin. 
Prolonged increased frequencies of CD4+CD25+ T cells after induction therapy with OKT3, followed by high-dose rapamycin and low dose tacrolimus after islet transplantation were also demonstrated. These cells showed better suppression of the in vitro proliferative response to donor cells than to third-party cells, suggesting the induction of donor directed Treg.

Campath-1H (Alemtuzumab) treatment in renal allograft recipients resulted in a significant increase in the number of CD4+CD25+FOXP3+ lymphocytes up to several years (28). In the peripheral blood of islet allograft recipients, Alemtuzumab was similarly associated with an early although transient increase in the frequency of CD4+CD25+FOXP3+ T cells compared with patients receiving Daclizumab or Thymoglobulin (29).

\section{Treg-based therapy in transplantation}

Much experimental evidence suggests that adoptive transfer of Treg can control transplant allograft rejection in rodents. By extension, such adoptive transfer of in vitro generated Treg may be applicable to clinical immunosuppression and tolerance protocols. However, in humans Treg-based treatment modalities may be hampered by several conditions. The low number of natural Treg necessitates in vitro expansion of cells using various culture conditions for polyclonal bulk cultures. General immune suppression might occur by using in vitro expanded polyclonal $\mathrm{CD} 4{ }^{+} \mathrm{CD} 25^{+}$Treg, as the ratio of antigen-specific Treg is typically very low in the population, resulting in low efficiency.

Adoptive transfer of antigen-specific Treg in mice can prevent allograft rejection with high efficiency, and does not compromise general immunity (30). Large scale in vitro generation and expansion of human alloantigen-specific Treg seems possible. Treg from type-1 diabetes mellitus patients and control individuals were isolated by cell sorting, and expanded in vitro after stimulation with anti-CD3/anti-CD28 coated microbeads and IL-2. Expansion of CD4+CD127low/-CD25+ T cells, particularly the CD45RA+ subset, resulted in functional Treg with sustained high FOXP3 and production of regulatory cytokines, suggesting that these cells offered a suitable cell population for cellular therapy in this autoimmune disease (10).

Optimal Treg therapy in humans should ideally use antigen-specific rather than polyclonal Treg. Dendritic cells have been used to expand alloantigen-specific CD25+ CD4+ Treg in the presence of IL-2. Allogeneic dendritic cells were more effective than spleen cells for expanding Treg suggested by high levels of FOXP3, and antigen-specific suppression in the mixed leukocyte reaction (31). When allogeneic, nontransformed B cells were used as stimulators, rapid induction and expansion of functional human alloantigen-specific Treg was reported after 3 weeks of culture without any exogenous cytokines. The resulting CD4+CD25highFOXP3+ alloantigen-specific Treg had cell-contact dependent suppressive function which partially relied on CTLA4 expression (32).

Recent observations in murine systems elucidate the in vivo regulatory mechanisms of antigen-specific Treg cells, and also underline the strengths and limitations of these cells as possible immunotherapy for solid organ transplantation. Antigen specific Treg, expanded in vitro, promote donor-specific transplantation tolerance in $\mathrm{T}$ cell depleted recipients. Treg can divide in vivo, migrate to the allograft and the draining lymph nodes. They prevent cellmediated graft rejection by controlling the differentiation of alloreactive CD4+ T cells into cytokine producing Th1 effector T cells, and by preventing the infiltration of naïve CD4+CD25- T cells into the allograft through modified expression of homing receptors. These Treg, however, have only limited regulatory effect in full MHC-mismatched models or in non-lymphopenic recipients (33). Extrapolation of these results to humans suggests Treg alone will have only modest suppressive effects in solid organ transplantation. 


\section{Summary and future directions}

At present, the diagnostic and predictive meaning and possible usefulness of FOXP3 levels in transplant patients remains uncertain. FOXP3 regulates $\mathrm{T}$ cell function in a dosedependent manner and sustained expression of FOXP3 in mature Treg is required to maintain the suppressive transcriptional program established during their commitment. Constant exposure to alloantigen after transplantation may be required to achieve sustained levels of FOXP3 expression and for Treg lineage stability. Higher levels of FOXP3 appear to be associated with reversible acute rejection, and lower levels with graft failure. Thus, quantitative analysis of individual cellular expression may provide a valuable monitoring tool for allograft rejection. The reported lack of correlation between peripheral and the local or intragraft FOXP3 levels, however, indicates that this gene may not be a potential candidate for non-invasive detection of non-responsiveness.

As for the relationship between immunosuppression and Treg, the results from both experimental and clinical studies show that the use of specific immunosuppression may be an essential component of strategies to induce and maintain transplant tolerance by Treg. Clinical studies show that immunosuppressive drugs can have detrimental or beneficial effects on Treg.

Treg are a promising approach to prevent acute and chronic rejection, and for induction of tolerance. To develop treatment strategies, establish dose and administration protocols, and to measure and monitor efficacy, however, fundamental gaps need to be filled in our knowledge. Finding a more definitive regulatory phenotype will be in the forefront of future efforts. Considering the facts that graft outcome partly depends on Treg/Teff ratios, and the frequency of allospecific Treg is probably much lower than those of Teff, interactions between Treg and immunosuppression will have to be further explored. Understanding the complex homeostasis of in vitro generation, expansion of antigen-specific human Treg, and developing adjuvant therapies for in vivo application still remains a major challenge. Developments in these areas will be crucial for safe and effective human use.

\section{Acknowledgments}

Funding sources: NIH RO1 AI062765

\section{References}

1. Scheffold A, Murphy KM, Höfer T. Competition for cytokines: Treg cells take all. Nat Immunol. 2007; 8(12):1285-1287. [PubMed: 18026078]

2. Wood KJ, Sakaguchi S, Regulatory T. cells in transplantation tolerance. Nat Rev Immunol. 2003; 3(3):199-210. [PubMed: 12658268]

3. Pandiyan P, Zheng L, Ishihara S, Reed J, Lenardo MJ. CD4+CD25+Foxp3+ regulatory T cells induce cytokine deprivation-mediated apoptosis of effector CD4+ T cells. Nat Immunol. 2007; 8(12):1353-1362. [PubMed: 17982458]

4. Ludwig-Portugall I, Hamilton-Williams EE, Gottschalk C, Kurts C. Cutting Edge: CD25+ regulatory $\mathrm{T}$ cells prevent expansion and induce apoptosis of $\mathrm{B}$ cells specific for tissue autoantigens. J Immunol. 2008; 181(7):4447-4451. [PubMed: 18802046]

5. Wang R, Wan Q, Kozhaya L, Fujii H, Unutmaz D. Identification of a regulatory T cell specific cell surface molecule that mediates suppressive signals and induces Foxp3 expression. PLoS ONE. 2008; 3(7):e2705. [PubMed: 18628982]

6. Liu W, Putnam AL, Xu-Yu Z, Szot GL, Lee MR, Zhu S. CD127 expression inversely correlates with FoxP3 and suppressive function of human CD4+ T reg cells. J Exp Med. 2006; 203(7):17011711. [PubMed: 16818678] 
7. Pillai V, Ortega SB, Wang CK, Karandikar NJ. Transient regulatory T-cells: a state attained by all activated human T-cells. Clin Immunol. 2007; 123(1):18-29. [PubMed: 17185041]

8. Wang J, Ioan-Facsinay A, van der Voort E, Huizinga TW, Toes RE. Transient expression of FOXP3 in human activated nonregulatory CD4 (+) T cells. Eur J Immunol. 2007; 37(1):129-138. [PubMed: 17154262]

9. Selvaraj RK, Geiger TL. A kinetic and dynamic analysis of Foxp3 induced in T cells by TGF-beta. J Immunol. 2007; 178(12):7667-7677. [PubMed: 17548603]

10. Putnam AL, Brusko TM, Lee MR, Liu W, Szot GL, Ghosh T, et al. Expansion of human regulatory T-cells from patients with type 1 diabetes. Diabetes. 2009; 58(3):652-662. [PubMed: 19074986]

11. Zheng Y, Josefowicz SZ, Kas A, Chu TT, Gavin MA, Rudensky AY. Genome-wide analysis of Foxp3 target genes in developing and mature regulatory T cells. Nature. 2007; 445(7130):936940. [PubMed: 17237761]

12. Koenen HJ, Smeets RL, Vink PM, Rijssen EV, Boots AM, Joosten I. Human CD25highFoxp3pos regulatory T-cells differentiate into IL-17 producing cells. Blood. 2008; 112(6):2340-2352. [PubMed: 18617638]

13. Voo KS, Wang YH, Santori FR, Boggiano C, Wang YH, Arima K, et al. Identification of IL-17producing FOXP3+ regulatory T cells in humans. Proc Natl Acad Sci U S A. 2009; 106(12):47934798. [PubMed: 19273860]

14. Tuovinen H, Laurinolli TT, Rossi LH, Pekkarinen PT, Mattila I, Arstila TP. Thymic production of human FOXP3(+) regulatory T cells is stable but does not correlate with peripheral FOXP3 expression. Immunol Lett. 2008; 117(2):146-153. [PubMed: 18321596]

15. Vukmanovic-Stejic M, Zhang Y, Cook JE, Fletcher JM, McQuaid A, Masters JE. Human CD4+ CD25hi Foxp3+ regulatory $\mathrm{T}$ cells are derived by rapid turnover of memory populations in vivo. J Clin Invest. 2006; 116(9):2423-2433. [PubMed: 16955142]

16. Akbar AN, Vukmanovic-Stejic M, Taams LS, Macallan D. The dynamic co-evolution of memory and regulatory CD4+ T cells in the periphery. Nat Rev Immunol. 2007; 7(3):231-237. [PubMed: 17318234]

17. Dijke IE, Caliskan K, Korevaar SS, Balk AH, Zondervan PE, Maat AP, et al. Acute rejection is associated with high FOXP3 mRNA expression levels in the graft, but not in the peripheral blood, of heart transplant recipients. J Heart Lung Transplant. 2007; 26(2):S72.

18. Muthukumar T, Dadhania D, Ding R. Messenger RNA for FOXP3 in the urine of renal-allograft recipient. N Engl J Med. 2005; 353(22):2342-2351. [PubMed: 16319383]

19. Bunnag S, Allanach K, Jhangrib GS, Sis B, Einecke G, Mengel M, et al. FOXP3 expression in human kidney transplant biopsies is associated with rejection and time post transplant but not with favorable outcomes. Am J Transplant. 2008; 8(7):1423-1433. [PubMed: 18510637]

20. Louis S, Braudeau C, Giral M, Dupont A, Moizant F, Robillard N, et al. Contrasting CD25hi $\mathrm{CD} 4+\mathrm{T}$ cells/FoxP3 patterns in chronic rejection and operational drug-free tolerance. Transplantation. 2006; 81(3):398-407. [PubMed: 16477227]

21. Koshiba T, Li Y, Takemura M, Wu Y, Sakaguchi S, Minato N, et al. Clinical, immunological, and pathological aspects of operational tolerance after pediatric living-donor liver transplantation. Transpl Immunol. 2007; 17(2):94-97. [PubMed: 17306739]

22. Li Y, Zhao X, Cheng D, Haga H, Tsuruyama T, Wood K, et al. The presence of Foxp3 expressing T cells within grafts of tolerant human liver transplant recipients. Transplantation. 2008; 86(12): 1837-1843. [PubMed: 19104431]

23. Demirkiran A, Baan CC, Kok A, Metselaar HJ, Tilanus HW, van der Laan LJ. Intrahepatic detection of FOXP3 gene expression after liver transplantation using minimally invasive aspiration biopsy. Transplantation. 2007; 83(6):819-823. [PubMed: 17414718]

24. Verdonk RC, Haagsma EB, Jonker MR, Bok LI, Zandvoort JH, Kleibeuker JH, et al. Effects of different immunosuppressive regimens on regulatory T-cells in noninflamed colon of liver transplant recipients. Inflamm Bowel Dis. 2007; 13(6):703-709. [PubMed: 17230494]

25. Vlad G, Ho EK, Vasilescu ER, Fan J, Liu Z, Cai JW, et al. Anti-CD25 treatment and FOXP3positive regulatory $T$ cells in heart transplantation. Transpl Immunol. 2007; 18(1):13-21. [PubMed: 17584597] 
26. Bluestone JA, Liu W, Yabu JM, Laszik ZG, Putnam A, Belingheri M, et al. The effect of costimulatory and interleukin 2 receptor blockade on regulatory $\mathrm{T}$ cells in renal transplantation. Am J Transplant. 2008; 8(10):2086-2096. [PubMed: 18828769]

27. Segundo DS, Ruiz JC, Izquierdo M, Velthuis J, Geel A, et al. Conversion to steroid free rapamycin, reduce percentages of $\mathrm{CD} 4+\mathrm{CD} 25$ highFoxP3+ T cells in renal transplant patients. Am J Transplant. 2007; 7:S508.

28. Bloom DD, Chang Z, Fechner JH, Dar W, Polster SP, Pascual J, et al. CD4+ CD25+ FOXP3+ regulatory $\mathrm{T}$ cells increase de novo in kidney transplant patients after immunodepletion with Campath-1H. Am J Transplant. 2008; 8(4):793-802. [PubMed: 18261176]

29. Toso C, Edgar R, Pawlick R, Emamaullee J, Merani S, Dinyari P, et al. Effect of different induction strategies on effector, regulatory and memory lymphocyte sub-populations in clinical islet transplantation. Transpl Int. 2009; 22(2):182-191. [PubMed: 18713144]

30. Joffre O, Santolaria T, Calise D, Al Saati T, Hudrisier D, Romagnoli P, et al. Prevention of acute and chronic allograft rejection with CD4+CD25+Foxp3+ regulatory T lymphocytes. Nat Med. 2008; 14(1):88-92. [PubMed: 18066074]

31. Yamazaki S, Inaba K, Tarbell KV, Steinman RM. Dendritic cells expand antigen-specific Foxp3+ CD25+ CD4+ regulatory T cells including suppressors of alloreactivity. Immunol Rev. 2006; 212:314-329. [PubMed: 16903923]

32. Tu W, Lau YL, Zheng J, Liu Y, Chan PL, Mao H, et al. Efficient generation of human alloantigenspecific CD4+ regulatory T cells from naive precursors by CD40-activated B cells. Blood. 2008; 112(6):2554-2562. [PubMed: 18599794]

33. Golshayan D, Wyss JC, Abulker CW, Schaefer SC, Lechler RI, Lehr HA, et al. Transplantation tolerance induced by regulatory T cells: In vivo mechanisms and sites of action. Int Immunopharmacol. 2009 Jan 9. 